\title{
A dynamic and automatic traffic light control system for solving the road congestion problem
}

\author{
W. Wen \& C. L. Yang \\ Department of Information Management, \\ LungHwa University of Science and Technology, \\ Taiwan, Republic of China \\ Department of Information Management, Tatung University, \\ Taiwan, Republic of China
}

\begin{abstract}
Traffic congestion is a severe problem in many modern cities all over the world. To solve the problem, we have designed a framework for a dynamic and automatic traffic light control system and developed a simulation model coded in Arena to help design the system. The model adopts average arrival time and departure times, which are physically observed at each intersection of Chung San North road, Taipei, Taiwan, to simulate the arrival and leaving number of cars during the run time period. In our experiment, by controlling light duration and speed limit, traffic congestion in a large city can be solved.
\end{abstract}

Keywords: simulation system; traffic light control system; traffic congestion.

\section{Introduction}

Traffic congestion has been causing many critical problems and challenges in most cities of modern countries. To the commuter or traveller, congestion means lost time, missed opportunities, and frustration. To the employer, congestion means lost worker productivity, trade opportunities, delivery delays, and increased costs. To solve congestion problems is feasible not only by physically constructing new facilities and policies but also by building information technology transportation management systems. A growing body of evidence proves that traffic congestion problems cannot be solved simply by expanding the road infrastructure. In fact, building new roads can actually compound congestion, in some cases, by inducing greater demand for vehicle travel - 
demand that quickly eats away the additional capacity. Therefore, many countries are working to manage their existing transportation systems to improve mobility, safety, and traffic flows and to reduce demand for vehicle use. By enhancing public transport, route guidance systems, traffic signal improvements, and incident management, congestion can be improved greatly. Of course, construction of new private bus ways, expressways, or subways to reduce this growth for easy travel has not kept pace. From recent analytical statistics [13], it is estimated that roughly half of the congestion is what is known as recurring congestion - caused by recurring demands that exist virtually every day, where road use exceeds existing capacity. The other half is due to non-recurring congestion caused by temporary disruptions. The four main reasons of nonrecurring congestion are: traffic incidents (ranging from disabled vehicles to major crashes), work zones, weather, and special events. Non-recurring events dramatically reduce available capacity and reliability of the entire transportation system. Researchers have done many researches to increase capacity and remove bottlenecks.

Schaefer et al. [11] developed a simulation model for evaluating freeway lane control signing. The simulation results show that lane control has little influence on congestion. However, the region between heavy and medium traffic flow is sensitive to lane control. Chen and Yang [3, 4] have created an algorithm to find the minimum total time path to simulate the operations of traffic-light control in a city. Stoilova and Stoilov [12] also built a simulation model to measure the best of traffic lights to achieve low noise levels with optimal traffic management and environmental pollution. Grau and Barcelo [5] and Messmerand and Papageorgiou [9] discussed the minimum of queue lengths in different intersections. To aid traffic management systems, Nooralahiyan et al. [10] adopted a Time Delay Neural Network (TDNN) to classify individual traveling vehicles based on their speed-independent acoustic signature. The TDNN network was trained and resulted in $94 \%$ accuracy for training patterns and $82.4 \%$ accuracy for test patterns. Besides, by adopting the shortest path algorithms, many other researches about solving traffic problems have been published [1, 2, 6-8, 15]. Wen and Hsu [14] designed a route navigation system with a new revised shortest-path routing algorithm and made a comparison of performance evaluation. Our research focuses on traffic signal improvements to solve part of traffic congestion problem, which will be discussed below.

\section{The framework for a dynamic and automatic traffic light control system}

The dynamic and automatic traffic light control system (DATLCS) is composed of seven elements: the radio frequency identification (RFID) reader, the active RFID tag, the personal digital assistance (PDA), the wireless network, the database, the knowledge base, and the backend server (see Figure 1). The RFID reader detects a RF-ACTIVE code at $1024 \mathrm{MHz}$ from the active tag pasted on the car. The active tag includes a battery so that it can periodically and actively transmit messages stored in the tag. Upon receiving the data, the reader will store 
all information in the PDA. After accumulating fix size of data, the PDA with a wireless card will connect to the backend server and store them into the database in the server. The server uses the data stored in the database to calculate maximum flow and average car speed. When all possible congestion roads and car speed are collected, these data will be used as the input parameters of the simulation model within the server. Using IF-THEN rule, the knowledge base provides different alternatives in terms of varieties of traffic situations. Upon getting the simulation results, the DTLCS is able to automatically set a red (green) light duration via the traffic light control interface for improving the traffic congestion problem.

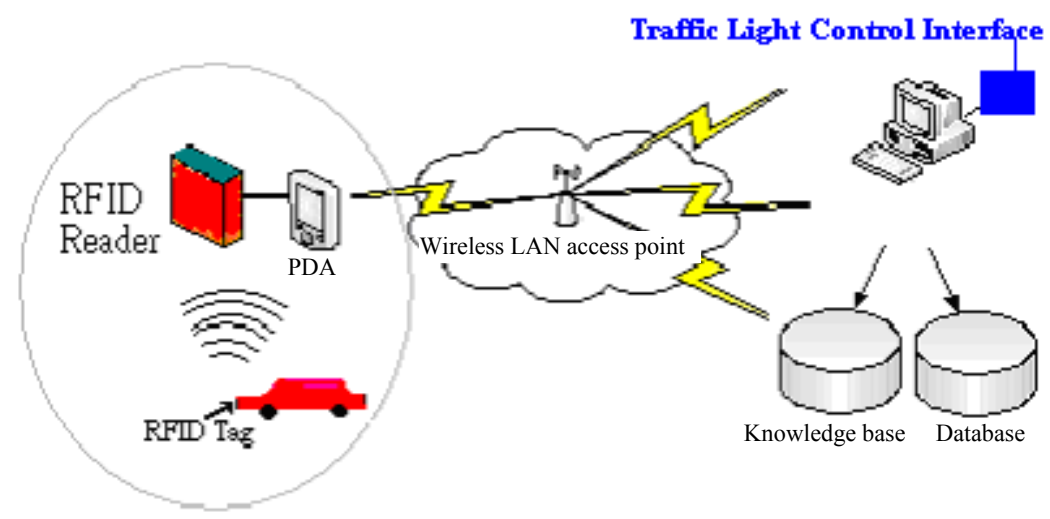

Figure 1: A framework for dynamic and automatic traffic light control systems.

\subsection{Definitions and notations of the simulation model}

To illustrate how the system works, we have developed a 3-intersection road traffic simulation model by using Arena. Before giving example, let us introduce the definition of notations as follows:

\section{Variables:}

T: The time for passing a length of a car.

N2: The remaining space of the second segment of a road, which is measured by the number of cars.

N3: The remaining space of the third segment of a road, which is measured by the number of cars.

cross 1: The time for a car passing the stop line at the first intersection.

cross 2: The time for a car passing the stop line at the second intersection.

cross 3: The time for a car passing the stop line at the third intersection.

clock light 1: Simulation clock time for traffic light signal at the first intersection turning to red.

clock light 2: Simulation clock time for traffic light signal at the second intersection turning to red.

clock light 3: Simulation clock time for traffic light signal at the third intersection turning to red. 
clock car 1: Simulation clock time of the car arriving at the stop line at the first intersection.

clock car 2: Simulation clock time of the car arriving at the stop line at the second intersection.

clock car 3: Simulation clock time of the car arriving at the stop line at the third intersection.

clock cross 1: Simulation clock time of the car permitting to leave the stop line at the first intersection pluses cross 1 .

clock cross 2: Simulation clock time of the car arriving at the stop line at the second intersection pluses cross 2.

clock cross 3: Simulation clock time of the car arriving at the stop line at the third intersection pluses cross 3 .

green 1:Green light duration at the first intersection.

red 1: Red light duration at the first intersection.

green 2: Green light duration at the second intersection.

red 2: Red light duration at the second intersection.

green 3: Green light duration at the third intersection.

red 3: Red light duration at the third intersection.

\section{Resources:}

switch 1: A resource for controlling traffic light signal or for determining whether a car permits to pass the stop line at the first intersection.

switch 2: A resource for controlling traffic light signal or for determining whether a car permits to pass the stop line at the second intersection.

switch 3: A resource for controlling traffic light signal or for determining whether a car permits to pass the stop line at the third intersection.

space2: The resources of cars capacity between the first intersection and the second intersection, which is used to determine whether there is enough space for a car driving to the second segment of a road.

space3: The resources of cars capacity between the second intersection and the third intersection, which is used to determine whether there is enough space for a car driving to the third segment of a road.

\section{Queues:}

departure1.Queue: A queue at the first intersection to store all waiting cars, which do not have rights to pass the intersection.

departure2.Queue: A queue at the second intersection to store all waiting cars, which do not have rights to pass the intersection.

departure3.Queue: A queue at the third intersection to store all waiting cars, which do not have rights to pass the intersection.

space2.Queue: A queue in the first segment of a road waiting for the space of the second segment of a road.

Space3.Queue: A queue in the second segment of a road waiting for the space of the third segment of a road. 


\subsection{Description of the simulation model}

First we choose Chung San North Road, Taipei, Taiwan, as our research target because it is always busy in the rush hours from $6 \mathrm{pm}$ to $8 \mathrm{pm}$. The model for simplicity only covers 3 intersections. In the road traffic simulation model shown as Figure 2, assumes that the road has 3 intersections and each one has a traffic light signal. In the model, interarrival time for each car is 1.72 seconds, departure time for a car passing the stop line at each intersection is 1.2 seconds, and the time for passing a length of a car is 0.41 seconds, which are physically observed in average by us. Each traffic light signal can be controlled by an entity named light control 1, light control 2, or light control 3. Besides, cars named entities are created based on cars' arrival time. Basically, entity, light control 1, 2, or 3, needs to seize a resource, switch 1, switch 2, or switch 3 for changing green light to red light at each intersection. Similarly, a car (i.e., an entity) wants to pass any intersection if only if the car seizes the resource. Without seizing the resource, switch 1, switch 2, or switch 3, the car or light control 1, 2, or 3 will be put in a queue and can do nothing but waiting. Therefore a car and light control compete with each other in order to get a resource. However, in order to let light control 1, light control 2, and light control 3 always get a resource, switch 1, switch 2, or switch 3, we set the highest priority for them and cars with lower priority. Furthermore, before a car seizes a resource, it needs to first check whether resource space2, the capacity of cars between the first intersection and the second intersection or resource space3, the capacity of cars between the second intersection and the third intersection, is available to allow the car to drive through.

It is worth noting that, in our model, we have considered a traffic light signal turning red at the period of a car driving through an intersection. Under this circumstance, when the current time of a car added the time passing the intersection is greater than the time of the traffic light at the intersection turning to red, it should be divided into two parts for allowing traffic light signal turning to red in due course and for the car continuing driving through. To achieve this purpose, some modifications have to be done are given below:

first part of the duration $=$ clock light $1-($ clock cross $1-$ cross 1$)+0.0001$ second part of the duration $=$ clock cross $1-$ clock light $1-0.0001$

What is more in our model, as a car leaves the stop line at an intersection, say intersection 1, and drives through to the second segment of the Chung San North road, the time for driving to that road is dynamic according to space left in the segment. The remaining space is measured by time. This equation will be:

the driving time of a car from the stop line at the first intersection to the last car of the queue of the second segment) $=\mathrm{T} * \mathrm{~N} 2$ 

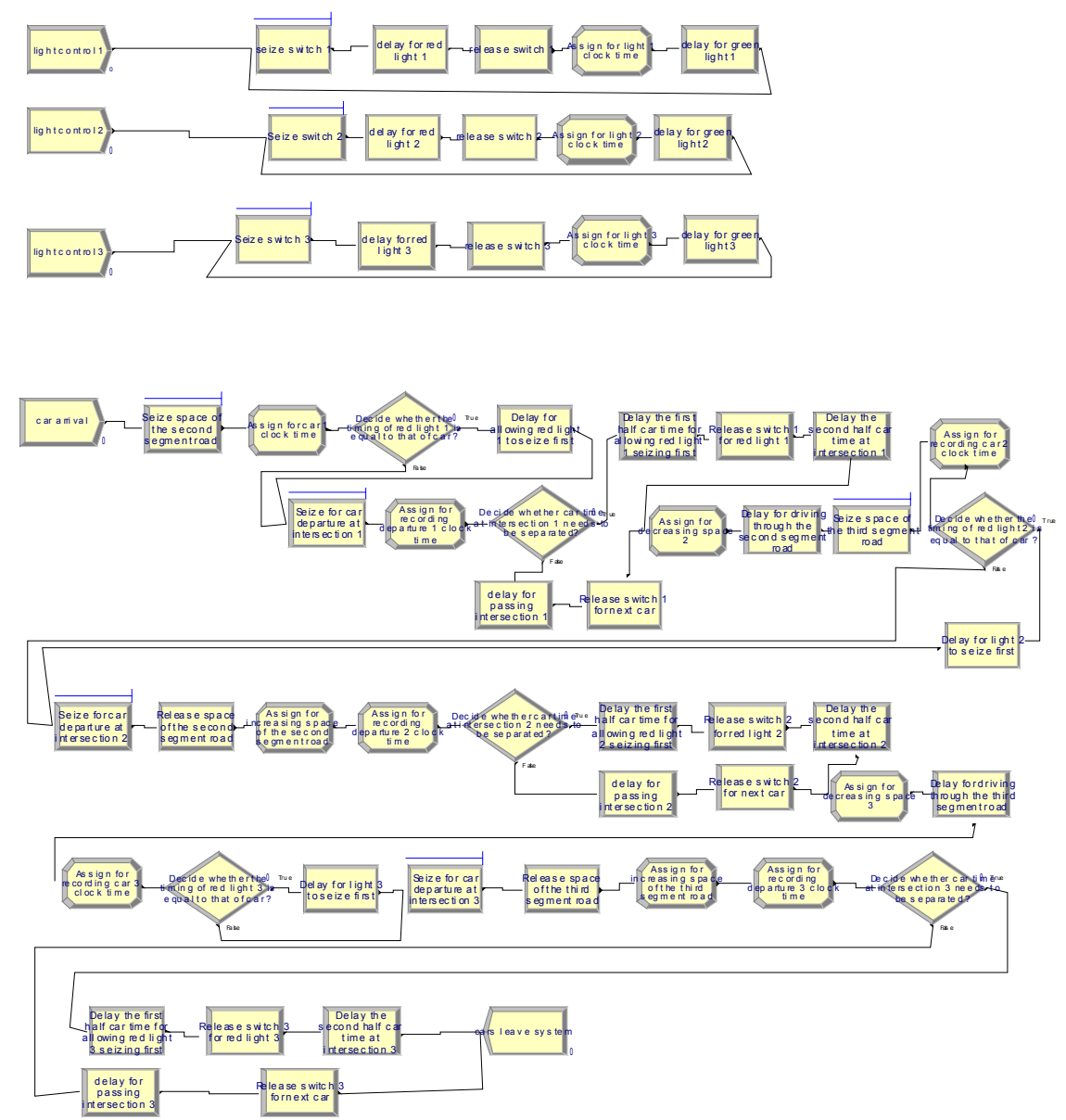

Figure 2: The traffic light control signal simulation model.

\section{Simulation analysis and results}

Using Arena, we have built a traffic light control signal simulation model. During the simulation analysis, initially a warm-up time of 1800 seconds for reaching system stable and a run time of 10,000 seconds are set. According to a practical measure, we fix the green light duration with 130 seconds. However, for the red light duration, it starts from 65 seconds and then is decreased by 5 seconds each time (i.e., 65, 60, 55, 50, 45, 40, 35, and 30). So totally we inspect and analyze 8 cases based on different red light durations.

Assume that the red light duration at each intersection is the same. The eight cases were modelled and run. Figure 3 presents when the red light duration is 50 seconds, the traffic light control model performs good performance. Although 
the average waiting time is still decreasing when the duration of the red light is less than 50 seconds, the time of decreasing is less than 5 seconds. At this circumstance, it doesn't seem worth reducing the red light duration by 5 seconds (e.g. 50 to 45). Particularly, if we reduce the red light duration at an intersection, it will decrease the green light duration in the other side at the same intersection, which will probably increase the average waiting time more than 5 seconds in that side.

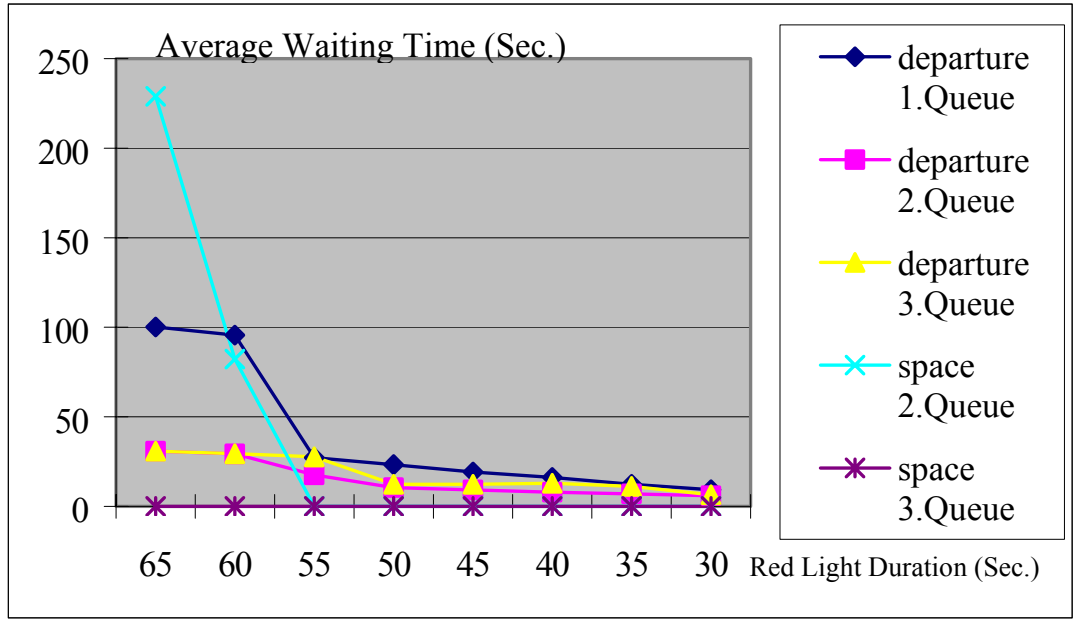

Figure 3: The average waiting time for different red light durations.

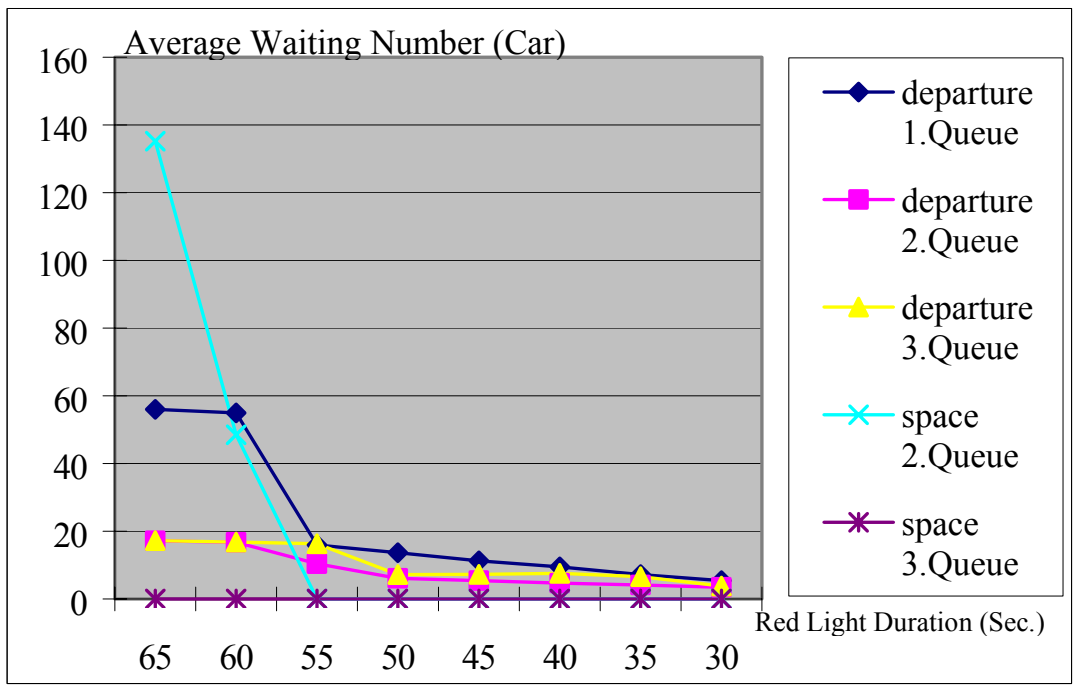

Figure 4: The average waiting number for different red light durations.

Similarly, in Figure 4 the average waiting number of cars at 50 seconds also has good performance. Figure 5 presents that the maximum waiting time of cars 
in space2.Queue is 454.8 seconds at the red light duration of 65 seconds, but will decrease to 0 at the duration of 50 seconds. In Figure 6 , the maximum number of cars in space2.Queue is 268 at the duration of 65 seconds. It cause the traffic system overcrowds at the first segment of the Chung San North road. To find out the best performance, we further divided the model into 8 categories based on the different combinations of red light duration at each intersection. The eight categories are $(\mathrm{r} 1=\mathrm{r} 2=\mathrm{r} 3=65),(\mathrm{r} 1=50 ; \mathrm{r} 2=\mathrm{r} 3=65),(\mathrm{r} 2=50 ; \mathrm{r} 1=\mathrm{r} 3=65)$, $(\mathrm{r} 3=50 ; \mathrm{r} 1=\mathrm{r} 2=65),(\mathrm{r} 1=\mathrm{r} 2=50 ; \mathrm{r} 3=65),(\mathrm{r} 1=\mathrm{r} 3=50 ; \mathrm{r} 2=65),(\mathrm{r} 2=\mathrm{r} 3=50 ; \mathrm{r} 1=65)$, and $(\mathrm{r} 1=\mathrm{r} 2=\mathrm{r} 3=50)$. Category 1 , for example, $\mathrm{r} 1=\mathrm{r} 2=\mathrm{r} 3=65$, means that the red light duration at intersections 1,2 and 3 are 65, 65, and 65 seconds respectively. The rest of categories can be explained in the same way. From Figure 7, we are able to know that the model for $r 1=r 2=r 3=50$ is the best alternative because the average waiting time of all queues at that situation is very low.

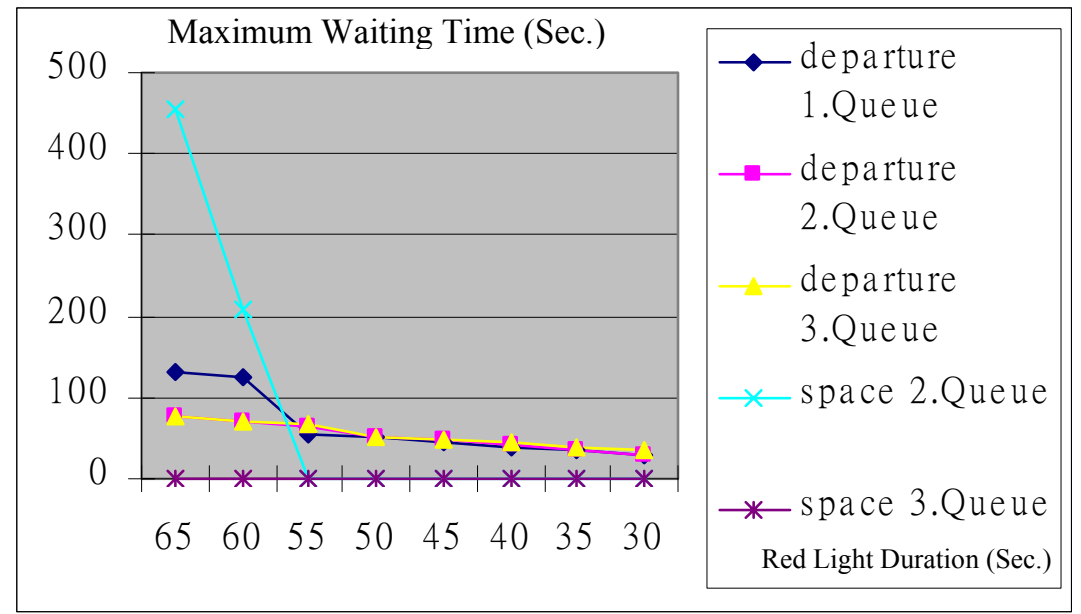

Figure 5: The maximum waiting time for different red light durations.

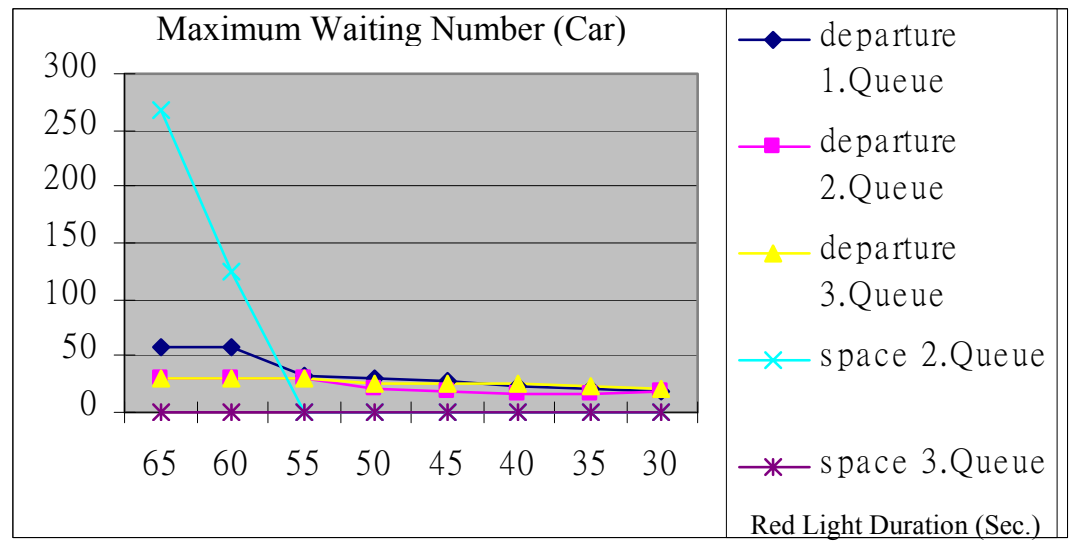

Figure 6: The maximum waiting number for different red light durations. 


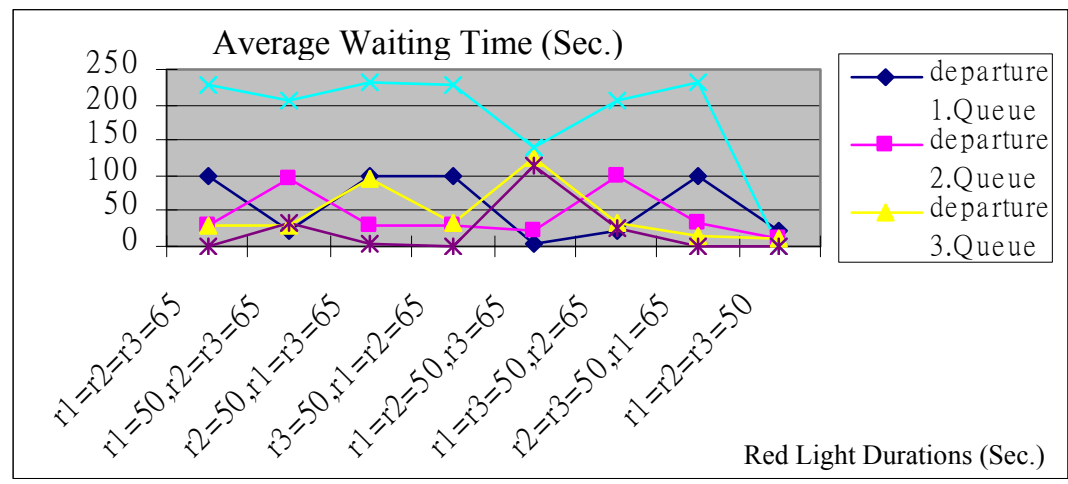

Figure 7: The average waiting time for various red light durations at different intersections.

Finally, it is worth mentioning that if we speed the time of cars departure, it also greatly reduces the average waiting time and average waiting number of cars. However, it is very difficult to force drivers to drive fast apart from increasing the speed limit.

\section{Conclusions and future work}

This paper proposes a new framework for dynamic and automatic traffic light control systems for improving traffic congestion problem. To know how to automatically and dynamically set the time duration of red (green) light signals, we also design a simulation model for improving traffic problem in rush hours according to the traffic conditions at the Chung San North road, Taipei, Taiwan. The simulation results physically prove the efficiency of the simulation model, because the average waiting time and number of cars are dropped down sharply when the red light duration is 50 seconds. Meanwhile, further analysis also shows if we set the red light durations of the three intersections are equal to 50 seconds, the total performance of the simulation model is the best.

Although this paper presents and analyzes the DATLCS, there are still several aspects where we can further improve its functions. In particular, we can extend the simulation model to add some more or different directions roads to let the model more close to the reality. In addition, because we can collect traffic flow and average car speed by using RFID technology, the method of dynamically finding a best route or a second optimal route for road navigation systems will be also a major research issue in the future.

\section{References}

[1] Chabini, I., Discrete Dynamic Shortest Path Problems in Transportation Applications, Transportation Research Record, 1998.

[2] Chabini, I., A New Algorithm for Shortest Paths in Discrete Dynamic Networks,8th IFAC/IFIP/IFORS Symposium on transportation systems, Tech Univ Crete, Greece, June 1997, pp. 16-18. 
[3] Chen, Y.L. and Yang, H.H. Minimization of Travel Time and Weighted Number of Stops in a Traffic-light Network, Transportation Research B, 34, 2000, pp. 241-253.

[4] Chen, Y.L. and Yang, H.H. Minimization of Travel Time and Weighted Number of Stops in a Traffic-light Network, European Journal of Operational Research, 144, 2003, pp. 565-580.

[5] Grau, R. and Barcelo, J., An Experience in Demand-responsive Traffic Control, Proceeding of 1st Meeting of the Euro Working Group in the Urban Traffic and Transportation, Landshut, Technical University of Munich, Germany, 1992.

[6] Ikeda, T., Hsu, M.Y. and Imai, H., A Fast A* Algorithm for Finding Better Routes by AI Search Techniques, Vehicle Navigation and Information Systems Conference Proceedings, IEEE, 1994, pp. 291-296.

[7] Ikeda, T. and Imai1, H., Fast A* Algorithms for Multiple Sequence Alignment. IPSJ SIG Notes 94-AL-42-7, IPSJ, 1994.

[8] Liu, B., Routing Finding by Using Knowledge about the Road Network, IEEE Transactions on System, man, and Cybernetics-Part A: Systems and Humans., Vol. 27, No. 4, pp. 425-430, July, 1997.

[9] Messmer, A., and Papageorgiou, M., Automatic Control Methods Applied to Freeway Network Traffic, 12 IFAC World Congress, Australia, Vol. 9, pp. 233-238.

[10] Nooralahiyan, A.Y., Dougherty, M., Mckeown, D., and Kirby, H.R., A Field Trail of Acoustic Signature Analysis for Vehicle Classification, Transport Research -C, Vol. 5, No. 3/4, pp. 165-177, 1997.

[11] Schaefer, L., Upchurch, J. and Ashur, S. A., An Evaluation of Freeway Lane Control Signing Using Computer Simulation, Math. Computer Modelling, Vol. 27, No. 9-11, pp. 177-187, 1998.

[12] Stoilova, K. and Stoilov T. Traffic Noise and Traffic Light Control, Transportation Research-D, Vol. 3, No. 6, pp. 399-417, 1998.

[13] US Department of transportation, Congestion Mitigation, http://www.fhwa.dot.gov/congestion/congest2.htm.

[14] W. Wen, and H. W. Hsu, A Route Navigation System with a New Revised Shortest-Path Routing Algorithm and Its Performance Evaluation, WIT Transactions on the Built Environment (Urban Transport), Vol. 77, pp. 733-743, 2005.

[15] Xia, L. and Shao, Y., Modelling of Traffic Flow and Air Pollution Emission with Application to Hong Kong Island, Environmental Modelling \& Software, 20, pp. 1175-1188, 2005. 